\title{
Órgano vomeronasal. Estudio anatómico de prevalencia y su función
}

\author{
The vomeronasal organ. Anatomic study of prevalence and its role \\ Sarría-Echegaray Pedro L¹, Artigas-Sapiaín Christian E1, Rama-López Julio, \\ Soler-Vilarrasa Ramona', Tomás-Barberán Manuel D'.
}

\begin{abstract}
RESUMEN
Introducción: El órgano vomeronasal (OVN) descrito por Jacobson en mamíferos distintos al ser humano, es una incógnita tanto en lo que se refiere a su localización así como a su función en la raza humana. Se considera como un vestigio del olfato, que en los animales mamíferos parece influir en los hábitos sexuales (feromonas) y sociales. Hasta la fecha han sido escasos los estudios concluyentes al respecto en humanos.

objetivo: Conocer la prevalencia del órgano vomeronasal en nuestras consultas.

Material y método: Presentamos un estudio prospectivo de prevalencia de la frecuencia de aparición de dicho órgano en 150 sujetos distribuidos por edad y sexo, explorados por endoscopia nasosinusal rígida. Por otro lado, analizamos la influencia sobre la libido (normal-disminuida-aumentada) en el posoperatorio de 35 septoplastías, a los 15 días tras retirada de taponamiento nasal y a los 30 días y lo comparamos con un grupo de 40 pacientes intervenidos timpanoplastías.

Resultados: Estudiados 150 sujetos, encontramos la presencia del órgano vomeronasal en el 39,33\% (59), de los cuales el 72,88\% (43) fue unilateral (23 derecha y 20 izquierda) y el 27,12\% (16) bilateral. En 91 (60,67\%) no hallamos dicha estructura. La libido de los 35 pacientes intervenidos de septoplastía estaba disminuida, a los 15 días, en el $77,14 \%$ (27) frente al $40 \%$ (16) de las timpanoplastías, normal en el 17,14\% (6) frente al 50\% (20) de las cirugías otológicas, y en 2 (5,7\%) poseptoplastía había aumentado, frente al $10 \%$ (4) del otro grupo. A los 30 días, en el 77,14\% (27) de las septoplastías se había normalizado frente al $90 \%$ (36) del grupo otológico, en 2 (5,71\%) de la cirugía nasal continuaba disminuida frente al 10\% (4) del grupo de las timpanoplastías y en $6(17,14 \%)$ tras septoplastía había aumentado. A todos los pacientes se les aplicó el mismo test no normalizado.

Conclusión: El órgano vomeronasal de Jacobson continúa siendo un gran desconocido. Es una estructura que, al parecer, no es constante, al menos a la exploración endoscópica nasosinusal. Es difícil valorar si la cirugía en sí misma o el trauma psicológico posquirúrgico son los que afectan la libido de los pacientes tras la cirugía.

Palabras clave: Órgano vomeronasal de Jacobson, libido, feromonas, septoplastía.
\end{abstract}

1 Servicio de Otorrinolaringología y Patología Cérvico-facial, Hospital Universitario Son Espases. Palma de Mallorca, España. 


\section{ABSTRACT}

Introduction: The vomeronasal organ (OVN) described by Jacobson in mammals other than humans is unknown both in terms of its location and its role in the human race. It is viewed as a vestige of smell, that mammals in the animal seems to influence the sexual habits (pheromone) and social. To date, few studies have been inconclusive on this in humans.

Aim: To determine the prevalence of vomeronasal organ in our medical consultations.

Materials and methods: We report a prospective prevalence study of the occurrence of such a body in 150 subjects distributed by age and sex explored by endoscopic sinus rigid. On the other hand, we analyze the effect on the libido (normally less-plus) in the postoperative 35 septoplasty, 15 days after the withdrawal of nasal pack and 30 days and compared with a group of 40 tympanoplasty surgery.

Results: Studied 150 subjects, we found the presence of the vomeronasal organ in $39.33 \%$ (59), of which $72.88 \%$ (43) had unilateral (23 right and 20 left) and 27.12\% (16) bilaterally. In 91 (60.67\%) did not find such a structure. The libido of the 35 patients who underwent septoplasty was decreased at 15 days, at $77.14 \%$ (27) versus $40 \%$ (16) of tympanoplasty, normal in $17.14 \%$ (6) compared to $50 \%$ (20) of otologic surgery, and in 2 (5.7\%) postseptoplasty had increased, compared to $10 \%$ (4) the other group. At 30 days, in $77.14 \%$ (27) of the septoplasty group the libido was normalized against $90 \%$ (36) in the otologic group. In 2 cases (5.71\%) of nasal surgery group was still decreased versus $10 \%$ (4) of cases of the tympanoplasty group, and in $6(17.14 \%)$ postseptoplasty was increased. All patients were administered the same test is not standardized.

Conclusion: The vomeronasal organ of Jacobson remains the great unknown. It is a structure that apparently is not constant, at least in the endoscopic sinus exploration. With regard to their role, it is difficult to assess whether the psychological trauma after surgery or the surgery by itself is responsible of the libido changes.

Key words: Vomeronasal organ of Jacobson, libido, pheromones, septoplasty.

\section{INTRODUCCIÓN}

Además del sentido del olfato, ¿tenemos la habilidad de percibir ciertas señales químicas emitidas por personas en nuestro entorno? Muchos otros mamíferos utilizan un conjunto separado de células receptoras quimiosensoriales, presentes en las fosas nasales, para percibir información social y sexual de los miembros de su propia especie, y existe la sospecha creciente de que nosotros también lo hacemos. Este órgano fue llamado órgano vomeronasal (OVN) u órgano de Jacobson. Ha sido muy estudiado en los mamíferos determinándose su estructura anatómica, la histología de las diferentes células que lo conforman, las vías neuronales que lo conectan con el bulbo olfatorio accesorio en el encéfalo y su función. Se ha intentado equiparar el OVN de los mamíferos con la estructura definida como tal en el ser humano, encontrándose similitudes y diferencias que llevan a dudar de su existencia y planean la incógnita de cuál es su verdadera función en el ser humano1.

Las sustancias químicas que es capaz de detectar el OVN son las llamadas feromonas. Su nombre deriva del griego pherein = transferir y de hormone = excitar. Su estructura química es sencilla y está compuesta por una parte volátil, el bisulfito de metilo, cuya función es la de provocar la atracción sexual; y una parte no volátil, la afrodisina, cuya función es provocar la monta del macho hámster al ser captada por su OVN. Estas feromonas son producidas por glándulas especializadas tanto en machos como en hembras, y acompañan las distintas secreciones de los individuos como saliva, sudor y secreciones vaginales. Algunos animales las detectan a muchos kilómetros de distancia. No sólo sirven para el contacto sexual de los individuos, sino también para demarcar el territorio y determinar la jerarquía en un grupo. Las feromonas no son detectadas exclusivamente por el OVN, sino también por el epitelio olfatorio principal². 
¿Los seres humanos tienen órganos vomeronasales? El primero que se planteó la función del OVN fue el anatomista danés, Ludwig Levin Jacobson, en 1811. La morfología del órgano ya se conocía, pero no su fisiología. En aquella época la fisiología era una ciencia muy marginal. Jacobson propuso que este órgano era una estructura sensorial muy especial que, quizás, tenía que ver con la percepción de olores singulares pero asumió que eran órganos no sensoriales, que aunque existían en embriones humanos, desaparecían durante el desarrollo o permanecían "vestigiales", es decir, desarrollados imperfectamente. Eso pasó más 0 menos desapercibido hasta que, ya al final del siglo XIX y principios del XX, Santiago Ramón y Cajal lo retomó.

Parece ser que el órgano de Jacobson, en los últimos tiempos denominado Par craneal 0 , es un órgano auxiliar del sentido del olfato en algunos vertebrados, los cuales son todos tetrápodos. Las neuronas bipolares sensoriales dentro del órgano detectan distintos compuestos químicos, habitualmente grandes moléculas llamadas feromonas. Las serpientes lo usan para oler presas, sacando la lengua y atrayendo partículas a la abertura del órgano en el paladar (conducto nasopalatino). Algunos mamíferos utilizan un movimiento facial característico llamado reflejo de Flehmen (del alemán flehmen - de animales, que significa arrugar el labio superior), es un tipo particular de movimiento de retracción en los labios en ungulados, félidos, y muchos otros mamíferos, para enviar compuestos a este órgano, mientras que en otros mamíferos la misma estructura se contrae, a través de grandes vasos sanguíneos que lo rodean y bombean para atraer los dichos compuestos químicos.

La mayoría de animales con un órgano vomeronasal lo utilizan para la detección de feromonas a pesar de que algunas de ellas son detectadas directamente por el sistema olfatorio tradicional. Las neuronas bipolares activadas transmiten a través de los nervios vomeronasales su información hasta el bulbo olfatorio accesorio, el cual tendría conexiones con el bulbo olfatorio principal, el sistema límbico y el hipotálamo, ejerciendo así sus funciones ${ }^{3}$.

Algunos científicos creen que en los humanos el órgano vomeronasal no es funcional como en el caso de otros animales, incluyendo cetáceos, algunos murciélagos y simios del nuevo mundo como los monos araña, monos capuchinos y monos ardilla. Estos científicos opinan también que en humanos adultos, no existe conexión entre el órgano y el cerebro ya que no se han encontrado claramente las neuronas bipolares, las vías neuronales que lo conecten al encéfalo ni el bulbo olfatorio accesorio en el cual acaban estas neuronas en los mamíferos que lo poseen. Sin embargo, existen evidencias en las que se sugiere que el órgano vomeronasal no se atrofia y permanece funcional durante toda la vida de una persona. Por tanto, su función en estos animales y humanos, si es real, es todavía un misterio.

El OVN se encuentra situado en el tercio anterior del tabique nasal (Figura 1) a unos 4-9,5 mm dorsal con respecto a la columella, a unos 6-12 $\mathrm{mm}$ del margen del orificio nasal externo ${ }^{3}$ y a 7 $\mathrm{mm}$ del suelo de la fosa nasal ${ }^{4}$. Son estructuras pares situadas a ambos lados del tabique nasal. Está conformado por una abertura vomeronasal de diámetro variable de 1-5 mm que se observa como una depresión con bordes sobreelevados en la mucosa del tabique nasal. Esta abertura se continúa a través de un conducto paralelo a los cartílagos paraseptales y al septum, que acaba en una dilatación tapizada con distintos tipos de células. Histológicamente, está formado por un epitelio respiratorio con cilios cortos que lo recubre y unas células llamadas receptoras, similares a neuronas bipolares ${ }^{5}$. No se han descubierto claras conexiones nerviosas entre el OVN y el encéfalo. La histología varía en función de la edad del individuo y se ve una rápida degeneración de ésta entre las

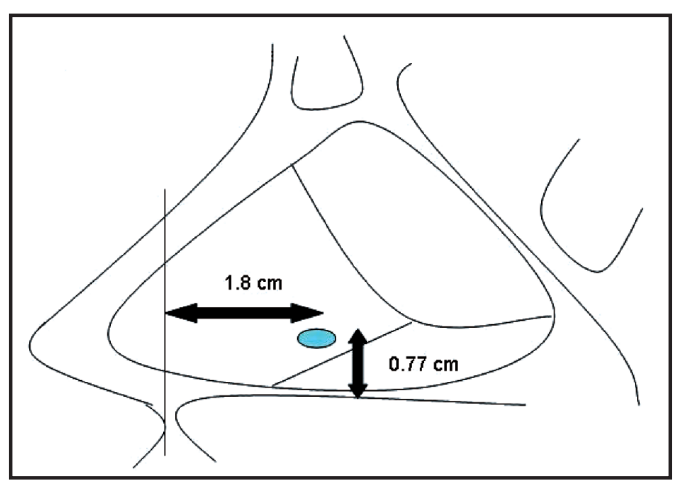

Figura 1. Ubicación anatómica del órgano vomeronasal ${ }^{12}$. (Modificado por C. Artigas-P Sarria). 
primeras semanas del período embrionario y el feto a término $0^{6,7}$.

"El OVN utiliza una diferente maquinaria molecular y parece ser una estructura mucho más primitiva que el sistema olfatorio principal", asevera Richard Axel, quien se ha ido interesando en este sistema. "Parece trabajar de una manera diferente, y nosotros no sabemos cómo",8,9.

Los OVNs están localizados en el septum nasal tomando su nombre del hueso vómer, donde el tabique se encuentra con el paladar duro. Al menos en roedores, las señales viajan desde el OVN hasta el bulbo olfatorio accesorio (antes que hasta el bulbo olfatorio principal) y luego, como lo mostró Sally Winans, de la Universidad de Michigan, en 1970, a partes del cerebro que controlan la reproducción y la conducta maternal. "Es una ruta alternativa hacia el cerebro", explica Rochelle Small, quien dirige el Programa de Sentidos Químicos, en el Instituto Nacional de la Sordera y Otros Trastornos de la Comunicación en Bethesda, en Maryland. Si el sistema olfatorio accesorio funciona en humanos como lo hace en roedores, evitando a la corteza cerebral, es probable que no tengamos ninguna consciencia de él en absoluto ${ }^{10}$.

\section{OBJETIVO}

El objetivo del presente estudio es conocer la prevalencia del órgano vomeronasal en pacientes que consultan en nuestro centro.

\section{MATERIAL Y MÉTODO}

Presentamos un estudio prospectivo de prevalencia de la presencia de dicho órgano en sujetos visitados en nuestras consultas. Como norma habitual, en las primeras visitas de la consulta de rinología, los pacientes son explorados de forma protocolizada mediante rinoscopía anteriory endoscopía nasosinusal. Se incluyeron 150 pacientes en los que se realizó una endoscopía nasosinusal (endoscopio rígido de $0^{\circ} \mathrm{y}$ 2,7 mm. de diámetro). Tan solo un 15\% precisaron anestesia tópica con vasoconstricción (ametocaína $1 \%$ con adrenalina a 1/1.000) en lentinas 0 algodón.

Se determinó, de forma visual la presencia o no de dicha estructura, unilateral o bilateral, mediante dicha técnica endoscópica.
Para evaluar la influencia sobre la libido, según la definición de la Real Academia Española de la Lengua, deseo sexual considerado por algunos autores como el impulso y raíz de las más variadas manifestaciones de la actividad psíquica (normaldisminuida-aumentada) en el posoperatorio, se preguntó a 46 intervenidos de septoplastías, a los 15 y 30 días tras retirada de taponamiento nasal, la siguiente pregunta: ¿Se han alterado sus hábitos 0 deseos sexuales tras la cirugía? (test no estandarizado ni normalizado). Partimos de la base hipotética que tras la realización de la técnica Cottle sobre el tabique nasal, la función del OVN se pudiera ver afectada. La misma pregunta se formuló, de forma contemporánea, a 40 intervenidos de timpanoplastía en el mismo intervalo de tiempo. Todos los pacientes fueron informados del motivo de las preguntas, dieron su consentimiento y dichas acciones fueron aprobadas por el Comité Ético de nuestra unidad, aceptando la responsabilidad definida por el Comité Internacional de Editores de Revistas Médicas (en www.icmje.org) y respetando las recomendaciones internacionales sobre investigación clínica (Declaración de Helsinki de la Asociación Médica Mundial en www.wma.net/e/policy).

Se realizó un análisis descriptivo de las variables, y se presentan las proporciones con su intervalo de confianza.

\section{RESULTADOS}

Estudiados 150 sujetos (Tabla 1), 82 varones $(54,7 \%)$ y 68 mujeres $(45,3 \%)$, con una media de edad de 46,3 años (16-76), encontramos la presencia del órgano vomeronasal en $59(39,3 \%)$, de Ios cuales $43(72,9 \%)$ fue unilateral ( 23 derecha y 20 izquierda) y en $16(27,1 \%)$ bilateral. En $91(60,7 \%)$ no hallamos dicha estructura (Figura 2).

En nuestro afán de valorar su función decidimos analizar la posible fluctuación de la libido en 46 pacientes sometidos a septoplastía. En 35 se pudo completar el estudio. Fueron 21 varones y 14 mujeres los incluidos, con una media de edad de 39,6 años (18-53) tras la realización de septoplastía tipo Cottle modificado, a los 15 y 30 días poscirugía. En el $77,1 \%$ (27) estaba disminuida, el 17,1\% (6) no refirió notar ningún tipo de variación al respecto y en 5,7\% (2) había aumentado a los 15 días tras la retirada de 
Tabla 1 Distribución edad-sexo

\begin{tabular}{|l|c|c|c|}
\hline Endoscopía nasal & Total 150 & \% del total & Edad media \\
\hline Hombres & 82 & 54,66 & 46 \\
\hline Mujeres & 68 & 45,34 & 43 \\
\hline
\end{tabular}

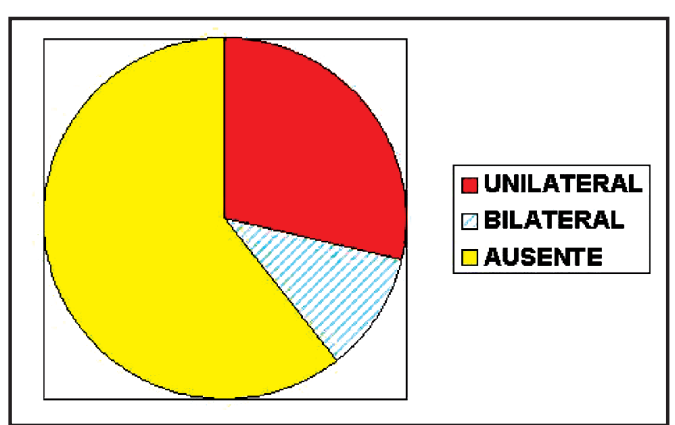

Figura 2. Presencia de OVN por endoscopía nasal.

taponamiento nasal, que habitualmente mantenemos durante 72 horas. Después de un mes de dicho acto, el $77,1 \%$ (27) consideraba que se había normalizado, el $5,7 \%$ (2) continuaba disminuida y el $17,1 \%$ (6) había aumentado. Todos los pacientes que refirieron aumento de la libido correspondían al sexo masculino (Figura 3). Con respecto a la influencia en las timpanoplastías en el $40 \%$ (16) estaba disminuida, el $50 \%$ (20) no refirió notar ningún tipo de variación al respecto y en el 10\% (4) había aumentado a los 15 días. Después de un mes de dicha intervención, el $90 \%$ (36) consideraba que se había normalizado, el $10 \%$ (4) continuaba disminuida y en ningún paciente refirió haber aumentado. Como anécdota decir que, dos de los pacientes pertenecientes al grupo que disminuyó la libido al mes de la timpanoplastía, daban un importante valor a los pabellones auriculares como zonas erógenas las cuales tras el abordaje retroauricular disminuyen la sensibilidad.

\section{DISCUSIÓN}

En los libros modernos de anatomía se ilustra la porción anterior del tabique nasal como tapizado por una mucosa lisa, sin observarse ninguna estructura 0 accidente en la misma. En cambio en los textos clásicos se puede observar la representación del OVN a ambos lados del tabique nasal'.

Los estudios anatómicos realizados en seres humanos sobre la presencia del OVN varían entre $28 \%$ y $100 \%$. Esto se debe probablemente a las contradicciones anatómicas existentes en la descripción del OVN y por el hecho de ser difícil de observar a la endoscopía nasal. También la prevalencia varía en función de si el estudio es endoscópico o se realiza un estudio histológico de las estructuras para determinar la presencia del OVN, o si el estudio es hecho en fetos, niños 0 adultos en distintas épocas de su vida.

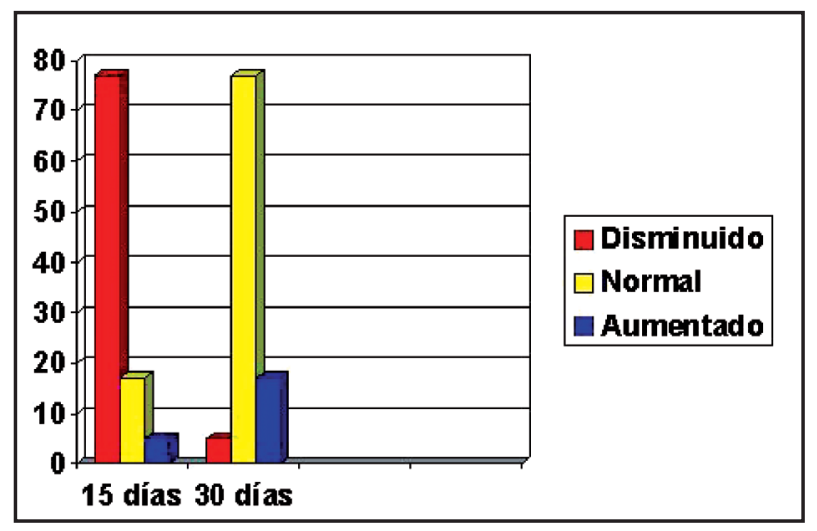

Figura 3. La libido tras la realización de septoplastía. 
García-Velasco y Mondragón ${ }^{11}$, en un estudio en 1.000 individuos, determinaron una frecuencia de $80 \%$ de OVN determinados con rinoscopía 0 endoscopía nasal si tenían un tabique nasal no desviado. Esta cifra aumentaba al $91 \%$ luego de haber corregido una desviación septal quirúrgicamente.

Más recientemente Zeller ${ }^{3}$, en un estudio realizado en 7 cadáveres fetales determinaron la existencia del OVN en el $100 \%$ de los fetos utilizando para ello endoscopía y estudios histológicos.

Won y cols ${ }^{4}$ tuvo hallazgos más similares a los nuestros en un estudio hecho en 22 cabezas de cadáveres en las cuales encontró $27,3 \%$ de OVN unilaterales, $31,8 \%$ OVN bilaterales y $40,9 \%$ sin OVN confirmado histológicamente. La ubicación y la forma del OVN fue igual a las descripciones previas agregando que se encontraba a alrededor de $0,77 \mathrm{~cm}$ del piso de la fosa nasal. El mismo autor prolongó el estudio a 80 pacientes vivos en los cuales encontró una frecuencia de $28,2 \%$ de existencia del OVN. Un hallazgo importante en su estudio fue que no todas las estructuras que a la visión endoscópica parecían ser el OVN, lo fueron al realizar el análisis histológico. Esto ha sido demostrado también por Bhatnagar y cols ${ }^{7}$ en un estudio con 31 cadáveres.

Gaafar $^{12}$ observó la presencia del OVN en el $76 \%$ de los casos sobre 200 sujetos estudiados, en forma de aberturas ovaladas o pequeñas depresiones. Acorde a los pocos y contradictorios estudios en humanos, solo algunos autores mencionan al conducto vomeronasal.

Mangakis ${ }^{13}$ observó un conducto vomeronasal de $62 \mathrm{~mm}$. Smith y cols ${ }^{10}$ refiere una longitud entre $3,5-11,8 \mathrm{~mm}$ y Eloit y cols ${ }^{11}$ observaron una Iongitud entre 2-5 mm.

Smith TD y cols investigaron en el órgano vomeronasal del humano adulto definiendo su localización, estructura y tamaño ${ }^{14}$. Por otro lado, hallamos referencias sobre su función en trabajos de Anton C y cols ${ }^{15}$.

Johson y cols ${ }^{16}$ lo encontraron en $39 \%$ de 100 pacientes estudiados endoscópicamente, Rapiejko y cols ${ }^{17}$ Io encontraron en $49,21 \%$ de 312 pacientes estudiados endoscópicamente y Besli y cols ${ }^{18}$ lo encontraron en $32 \%$ de los pacientes.

Finalmente Abolmaali y cols ${ }^{19}$ concluyen en un estudio realizado con resonancia nuclear magnética que el conducto vomeronasal tiene una longitud de $7 \mathrm{~mm}$, sufriendo numerosas variaciones en su tamaño y puede cruzar al lado contralateral.

Bellairs ${ }^{20}$ sostiene que los conductos están constituidos por cordones sólidos, que luego se canalizan 0 retrogradan para dar lugar al epitelio; la impermeabilidad completa en uno 0 ambos lados puede ocurrir, pero es rara en embriones y neonatos. Noyes examinó cortes seriados del tercio inferior de la cavidad nasal y de la región premaxilar de neonatos y halló en todos los casos analizados, pero no pudo comprobar la solución de continuidad del conducto nasopalatino entre la cavidad oral y la nasal ${ }^{21}$. Controversias en la literatura imposibilitan obtener una conclusión rigurosa con respecto a la permeabilidad del conducto nasopalatino en fetos y neonatos humanos.

La evidencia actual en humanos adultos lo determina como un órgano vestigial. La existencia de un OVN en humanos es interesante, debido a que ambos conductos cumplen con un rol fisiológico importante en el sistema vomeronasal de otros mamíferos, incluyendo algunos primates ${ }^{22}$.

En relación a la existencia de las feromonas humanas, se han realizado muchos estudios buscando estas sustancias químicas y varios de ellos han demostrado su existencia en secreciones vaginales y el sudor. Se ha visto que estas sustancias son capaces de producir una mayor atracción sexual entre hombres y mujeres y de sincronizar la menstruación en mujeres que conviven juntas ${ }^{23}$. Muchos autores se resisten a llamarlas feromonas y no hay acuerdo aún. Lo que sí esto demostraría es que estas sustancias químicas serían detectadas a través del epitelio olfatorio tradicional ya que nuestro OVN no sería funcionante 0 no se ha demostrado aún su función ${ }^{24}$.

Queremos hacer constar una serie de reflexiones que pudieran influir a modo de sesgos en los resultados y conclusiones de nuestro trabajo.

No hemos encontrado en la literatura cuestionarios contrastados referentes a la valoración de la libido que añadirían valor científico a nuestras conclusiones, ni tampoco se ha considerado el sexo de quién realizaba la pregunta y el sexo de quién era preguntado, lo cual pudiera influir en la fiabilidad de la respuesta. También nos podíamos haber planteado pasar el formulario antes y después de la intervención, pero supusimos que antes, todo era normal o la situación. No se valoró 
si la entrevista se realizaba a solas con el paciente 0 en presencia de algún familiar. La pregunta era realizada por el cirujano/a, a cargo, explicándole el porqué de la misma de la forma más sencilla y natural, sin ahondar en detalles.

No se consideró la presencia o no, macroscópica del OVN en los grupos entrevistados debido a que pudiéramos tener vestigios del mismo y solo ser capaces de apreciarlos con métodos de magnificación.

No se tuvo en cuenta el lado de la intervención quirúrgica en el caso de la cirugía otológica, ni la presencia o no del OVN, por los motivos anteriormente comentados.

Desconocemos el tipo de repercusión que pudiera tener sobre estas sensaciones el bloqueo con la consiguiente insuficiencia ventilatoria secundaria a la manipulación endonasal. El ser intervenido de la nariz tras dos semanas, presenta más repercusión que el ser operado del oído, 77,1\% frente al $40 \%$, con un intervalo de confianza del $95 \%$.

Por último considerar que las variables edad y sexo, dependerán de lo que cada uno considere hábitos 0 tendencias normales 0 alteradas, independientemente de la influencia de las mismas, sin desestimar que como todo órgano, estará sujeto a cambios, evoluciones e involuciones, en relación con la influencia de dichas variables.

\section{CONCLUSIÓN}

El órgano vomeronasal de Jacobson continúa siendo un gran desconocido en la raza humana. Es una estructura que al parecer no es constante, al menos a la exploración endoscópica nasosinusal, lo cual no implica que bajo control microscópico y estudio anatomopatológico lo sea.

La comunicación química comprende un amplio espectro de funciones en los mamíferos, que van desde el marcaje territorial hasta la elección de una pareja.

Las feromonas resultan ser sustancias importantes en la comunicación química entre los individuos de la misma especie.

A pesar de que en algunos mamíferos se ha comprobado que la percepción de estas sustancias altera la conducta y la fisiología del individuo, actualmente se discute su funcionalidad en los humanos.
Con respecto a su función, es difícil valorar si el trauma psicológico posquirúrgico es el máximo responsable de los cambios observados en la libido.

El hecho de que durante mucho tiempo se haya considerado que el órgano vomeronasal en los humanos era vestigial, y pese que en la actualidad haya trabajos que muestren que puede llegar a ser funcional, no puede descartar absolutamente, la presencia de un órgano vomeronasal funcional en las demás especies de primates.

Hemos de considerar que al no ser capaces de valorar su destrucción parcial o total tras el acto quirúrgico así como su posible reinervación, precisaremos de más estudios que nos ayuden a extraer conclusiones más concretas y rigurosas.

Queda una importante ventana abierta para el estudio de la prevalencia del OVN ampliando su presencia a nivel microscópico en el cadáver y buscar vías que permitan analizar y estudiar su supuesta función.

\section{BIBLIOGRAFÍA}

1. Borgarell MP. Aporte para el conocimiento anátomo-funcional del órgano vomeronasal humano y su probable relación con la conducta socio-sexual. Alcmeon, Revista Argentina de Clínica Neuropsiquiátrica 2007; 14: 5-48.

2. Singer AG, Acosta WC, Clancy an, Macrides $F$. The chemistry of vomeronasally detected pheromones: Characterization of an aphrodisiac protein. Ann New York 1987; 519: 287-98.

3. ZeLLER F. Anatomía normal y frecuencia del órgano vomeronasal de Jacobson en fetos humanos. Rev Argentina de Urología 2007; 72: 17-9.

4. Won J. The vomeronasal organ: An objective anatomic analysis of its prevalence. ENT-Ear, Nose and Throat Journal 2000; 79: 600-5.

5. SMith TD, Bhatnagar KP. The human vomeronasal organ. Part II: prenatal development. J Anat 2000; 197: 421-36.

6. Sмith TD, Bhatnagar KP. The human vomeronasal organ. Part III. Postnatal development from infancy to the ninth decade. J Anat 2001; 199: 289-302.

7. Smith TD, Bhatnagar KP. The human vomeronasal organ. Part IV: Incidence, topography, endoscopy, 
and ultrastructure of the nasopalatine recess, nasopalatine fossa, and vomeronasal organ. American Journal of Rhinology 2002; 16(6): 343-50.

8. Axel R, Dulac C. Expression of Candidate Pheromone Receptor Genes in Vomeronasal Neurons. Chem Senses 1998; 23: 467-75.

9. AXEL R. The molecular logic of smell. Sci Amer 1995; 273: 154-9.

10. Small RK, Leonard CM. Early recovery of function after olfactory tract section correlated with reinnervation of olfactory tubercle. Developmental Brain Research 1983; 7(1): 25-40.

11. García-Velasco J, Mondragón M. The incidence of the vomeronasal organ in 1000 human subjects and its possible clinical significance. J Steroid Biochem Molec Biol 1991; 39: 561-3.

12. Gaafar HA, Tantawy AA, Melis AA. The vomeronasal organ in adult humans: frequency of occurrence and enzymatic study. Acta Otolaryngol 1998; 118(3): 409-12.

13. Mangakis H. Ein Fall von Jacobson schen Organen beim Erwachsenen. Anat Anz 1906; 21: 106.

14. Smith TD, Siegel MI, Burrows AM. Searching for the vomeronasal organ of adult human: preliminary findings on locat ion, structure and size. Microsc Res Tech 1998; 15: 483.

15. Anton C, Wassef M, Ferrand J. Observations on adult human vomeronasal organs. Chem Senses 1988; (24): 64-8.
16. Johson A, Josephson R. Clinical and histological evidence for the presence of the vomeronasal organ in adult humans. J Otolaryngol 1985; 14(2): 71-9.

17. Rapiejko P, Zielnik-Jurkiewicz B. The existence vomeronasal organ in adult human. Otolaryngol Pol 2007; 61(4): 581-4.

18. BesLIR, SAYLAM C. The existence of the vomeronasal organ in human beings. J Craniofac Surg 2004; 15(5): $730-5$.

19. Abolmaali ND, Kühnau D. Imaging of the vomeronasal duct. Chem Senses 2001; (26): 35-9.

20. Bellairs Ad'A. Observations on the incisive canaliculi and nasopalatine ducts. $\mathrm{Br}$ Dent $\mathrm{J}$ 1951; 91: 281-6.

21. Noyes HJ. Nasopalatine Duct and Jacobson's organ in new-born infants. J Dent Res 1935; 15 : 155-6.

22. Hunter AJ, Fleming. The structure of the vomeronasal organ and nasopalatine ducts in Aotus trivirgatus and some other primate species. J Anat 1984; 138: 217-25.

23. SteRn K. MCcLintock M. Regulation of ovulation by humans pheromones. Nature 1998; 12: 177-9.

24. Barni T, MagGi M, Fantoni G. Sex oids and odorants modulate gonadotropin-releasing hormone secretion in primary cultures of human olfactory cells. J Clin Endocrinol \& Metab 1999; 84: 4266-73.

\footnotetext{
Dirección: Pedro L. Sarría-Echegaray

Carretera Valldemossa 79.

Secretaria ORL Son Espases Planta 0 - Módulo D

07120 Palma de Mallorca, España

Telef: 871-205363 / 205892

E mail: pedrosarria@orlmallorca.com
} 